\title{
On the stability and Hopf-bifurcation of a multi-delayed competitive population system affected by toxic substances with imprecise biological parameters
}

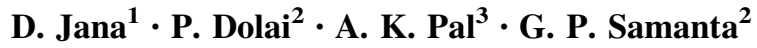

Received: 20 May 2016/Accepted: 4 June 2016/Published online: 20 June 2016

(C) Springer International Publishing Switzerland 2016

\begin{abstract}
In this paper we have analyzed the stability and Hopf-bifurcation behaviors of a multi-delayed two-species competitive system affected by toxic substances with imprecise biological parameters. We have exercised a method to handle these imprecise biological parameters by using parametric form of interval numbers. We have studied the feasibility of various equilibrium points and their stability. In case of toxic stimulatory system, the delay model exhibits a stable limit cycle oscillation due to variation in the delay parameters which lead to Hopf-bifurcation. Numerical simulations with a hypothetical set of data have been done to support the analytical findings.
\end{abstract}

Keywords Competing species - Imprecise parameters · Interval number $\cdot$ Multiple-delay $\cdot$ Hopf-bifurcation

G. P. Samanta

gpsamanta@math.iiests.ac.in; g_p_samanta@yahoo.co.uk

D. Jana

debaldevjana.jana@gmail.com

P. Dolai

pralay_dolai@yahoo.in

A. K. Pal

akpal_2002@yahoo.co.in

1 Department of Zoology, Ecological Modelling Laboratory, Visva Bharati University, Santiniketan 731235, India

2 Department of Mathematics, Indian Institute of Engineering Science and Techology, Shibpur, Howrah 711103, India

3 Department of Mathematics, S. A. Jaipuria College, Kolkata 700005, India

\section{Introduction}

Environmental pollution has emerged with it adversities to the modern civilization dwarfing the advantages to such a great extent of technological achievements. Since, industrialization contributes a lot to the economy of a nation, a massive resource is being invested in national industrialization. As a consequence, the industrial discharges contribute to a huge amount of contamination to the both aquatic and terrestrial environmental components (Jensen and Marshall 1982; Nelson 1970). A large amount of toxicant in the discharge makes it very difficult for that much species to exist on this earth. The poison inclines in the discharges, as a consequence, compels many species to go into extinction. Therefore, a circumstantial query is needed to put into the nature and activity of the discharged particles to combat with the situation and to our environment from these toxicant.

Mathematical models, here, can play a vital role to measure and minimize the aggression of the toxic elements in the environment and draw a sustainable atmosphere. The deterministic dynamic models with the effect of toxic substances on various ecosystems is studied by Hallam et al. (1983a, b), Hallam and Luna (1984), Luna and Hallam (1987), Freedman and Shukla (1991), Ghosh et al. (2002), He and Wang (2007), Das et al. (2009) and many others. Researchers, in course of their study over the effect and nature of the toxic elements come to an important observation that the enhanced population of one species might affect or control the growth of another or several other species through the production of allelopathic toxins or stimulants, thus influencing seasonal succession (Rice 1984). Unicellular green alga, chlorella Vulgaris can control the growth of their population and inhibit the growth of planktonic algae Asterionella formosa and 
Nitzschia frustrum (Bacillareae) with the toxin produced by themselves (Pratt 1940; Pratt and Fong 1940; Rice 1954). Toxic inhibition of phytoplankton by other phytoplankton has been observed by several researchers. In addition to that, it is also observed that some of the algae produce auxin that enhances the stimulation towards the growth of the other algae (Anderson 1989). These types of allelopathic stimulators and inhibitors play deciding role in moulding algal succession, blooms and pulses by creating or causing stimulated (inhibited) species to have a selective advantage or disadvantage in question of existence (Rice 1984; Berglund 1969).

In reality, time delays occur in almost every ecological situation and assume to be most of the reasons of regular fluctuations on population biomass. Kuang (1993) mentions that animals must take time to digest their food before further activities and responses take place and hence any model of species dynamics without delays is an approximation at best. Therefore, time delays can be included in the mathematical population model due to various ways such as maturation time, capturing time and other reasons. Moreover, existence of time delays is frequently a source of instability in some way. To make a more realistic biological model, many researchers (Maiti et al. 2008, 2008; Celik 2008; Gopalsamy 1992; MacDonald 1989; Xua et al. 2009; Liu 1994; Chen et al. 2007; Song et al. 2004) have incorporated time delay in their respective models.

Several researchers in theoretical ecology have considered their models based on the assumption that the biological parameters are precisely known. But in reality, the values of all the biological parameters can not be determined precisely for the lack of information and data, mistakes in the measurement process and determining the initial conditions. To overcome these difficulties in the field of mathematical biology, imprecise model is more realistic. Environmental fluctuations or imprecise biological phenomenon are the important causes of the impreciseness of the bio-mathematical model. There are many approaches to handle such models with imprecise parameters such as stochastic approach, fuzzy approach, fuzzy stochastic approach etc. In stochastic approach the imprecise parameters are replaced by random variables with known probability distributions. In fuzzy approach the imprecise parameters are replaced by fuzzy sets with known membership functions. In fuzzy stochastic approach some parameters are as fuzzy in nature and rest of the parameters are taken as random variables. However it is very difficult to draw a suitable membership function or a suitable probability distribution for each of the imprecise biological parameters. Recently researchers have introduced fuzzy models in prey-predator system such as Bassanezi et al. Bassanezi et al. (2000), Peixoto et al. Peixoto et al. (2008) and Guo et al. Guo et al. (2003), etc. Pal et al.
(2012, (2014, (2013) have considered an optimal harvesting prey-predator system with imprecise biological parameters and they have also discussed bio-economic equilibrium and optimal harvesting policy.

In this paper, we have introduced a multi-delayed two species competitive system which is affected by toxicant. To make the delay model more realistic, we have considered imprecise biological parameters as interval numbers. We present the interval numbers in parametric function form and study the parametric model Maynard-Smith (1975). The dynamical behavior of the parametric model is investigated for several values of the parameter $p \in[0,1]$. In Sect. 2, we discuss some basic definitions on interval numbers. In Sect. 3, we represent mathematical form of two species competitive system with time delays. Section 4 briefly states the delayed two species competitive system with imprecise parameters. Then parametric form of the systems is formulated to study the different behaviors of the model. Section 5 deals with the equilibrium points of the systems, their existence and stability analysis. Our important analytical results are numerically verified in Sect. 6. Finally, Sect. 7 contains the general discussion of the paper.

\section{Basic definitions}

In this section we discuss some basic definitions of the interval number and interval-valued function which have been used to study the imprecise competition model.

Definition 1 (Interval number) An interval number $A$ is represented by closed interval $\left[a_{l}, a_{u}\right]$ and defined by $A=\left[a_{l}, a_{u}\right]=\left\{x: a_{l} \leq x \leq a_{u}, x \in \mathfrak{R}\right\}$, where $\mathfrak{R}$ is the set of real numbers and $a_{l}, a_{u}$ are the lower and upper limits of the interval number, respectively.

So, every real number can also be presented by the interval number $[a, a]$, for all $a \in \mathfrak{R}$.

Definition 2 (Interval-valued function) Let us consider the interval $[a, b]$ where $a>0$. The interval $[a, b]$ can be expressed as a function $\phi(p)=a^{(1-p)} b^{p}$ for $p \in[0,1]$. This function is called interval-valued function.

Now we present some arithmetic operations on intervalvalued functions. Let $A=\left[a_{l}, a_{u}\right]$ and $B=\left[b_{l}, b_{u}\right]$ be two interval numbers, then

Addition $\quad A+B=\left[a_{l}, a_{u}\right]+\left[b_{l}, b_{u}\right]=\left[a_{l}+b_{l}, a_{u}+b_{u}\right]$. The interval-valued function for the interval $A+B$ is given by $\phi(p)=a_{L}^{(1-p)} a_{U}^{p}$ where $a_{L}=a_{l}+b_{l}$ and $a_{U}=a_{u}+b_{u}$. Subtraction $A-B=\left[a_{l}, a_{u}\right]-\left[b_{l}, b_{u}\right]=\left[a_{l}-b_{u}, a_{u}-b_{l}\right]$, provided $a_{l}-b_{u}>0$. The interval-valued function for the interval $A-B$ is given by $\phi(p)=b_{L}^{(1-p)} b_{U}^{p}$ where $b_{L}=$ $a_{l}-b_{u}$ and $b_{U}=a_{u}-b_{l}$. 


Scalar $\quad$ multiplication $\quad \kappa A=\kappa\left[a_{l}, a_{u}\right]=$
$\left\{\begin{array}{ll}{\left[\kappa a_{l}, \kappa a_{u}\right],} & \text { if } \kappa \geq 0 \\ {\left[\kappa a_{u}, \kappa a_{l}\right],} & \text { if } \kappa<0\end{array}\right.$, provided $a_{l}>0$. The interval-
valued function for the interval $\kappa A$ is given by $\phi(p)=$
$\left\{\begin{array}{cc}c_{L}^{(1-p)} c_{U}^{p} & \text { if } \kappa \geq 0 \\ -d_{U}^{(1-p)} d_{L}^{p} & \text { if } \kappa<0\end{array}, \quad\right.$ where $\quad c_{L}=\kappa a_{l}, \quad c_{U}=$
$\kappa a_{u}, \quad d_{L}=|\kappa| a_{l}, \quad d_{U}=|\kappa| a_{u}$.

\section{Basic mathematical model}

Maynard-Smith (1975) have considered a two species competing system:

$$
\begin{aligned}
& \frac{d N_{1}}{d t}=N_{1}\left[K_{1}-\alpha_{1} N_{1}-\beta_{12} N_{2}-\gamma_{1} N_{1} N_{2}\right], \\
& \frac{d N_{2}}{d t}=N_{2}\left[K_{2}-\alpha_{2} N_{2}-\beta_{21} N_{1}-\gamma_{2} N_{1} N_{2}\right],
\end{aligned}
$$

with initial data

$$
N_{1}(0) \geq 0, N_{2}(0) \geq 0 .
$$

Here $N_{1}(t), N_{2}(t)$ denote the population biomass of two competing species at time $t$, having common food resources; $K_{1}, K_{2}, \alpha_{1}, \alpha_{2}, \beta_{12}, \beta_{21}, \gamma_{1}, \gamma_{2}$ are positive constants. Here $K_{i}$ is the intrinsic growth rate of species $i, \alpha_{i}$ represents the interspecies competition coefficient of species $i$, $\beta_{i j}$ denotes the inter-species competition rate of $j$ th species upon the $i$ th species and $\gamma_{i}$ denotes the toxic inhibition rate of the $i$ th species $(i \neq j, \quad i, j=1,2)$. Samanta (2010) also analyze dynamical behaviors of such model where a toxic substance is produced at a constant rate. When the toxic coefficients $\gamma_{i}>0 \quad(i=1,2)$, the model (1) represents toxic inhibited species system. If we assume $\gamma_{i}=-\gamma_{i}^{\prime} \quad(i=1,2)$, where $\gamma_{i}^{\prime}>0$, then the model 1 represents toxic stimulated system. We assume that each species produces a substance toxic to the other, but only when the other is present. Further, it is also reasonable to assume such model where a toxic substance is produced to the competing species will not be instantaneous and takes discrete time lag which is regarded as maturity period of the species. Here we introduce the time lag $\tau_{i}(i=1,2)$ for the maturity of the species $N_{i}(i=1,2)$. Then the system 1 reduces to

$$
\begin{aligned}
& \frac{d N_{1}}{d t}=N_{1}\left[K_{1}-\alpha_{1} N_{1}-\beta_{12} N_{2}-\gamma_{1} N_{1} N_{2}\left(t-\tau_{2}\right)\right], \\
& \frac{d N_{2}}{d t}=N_{2}\left[K_{2}-\alpha_{2} N_{2}-\beta_{21} N_{1}-\gamma_{2} N_{1}\left(t-\tau_{1}\right) N_{2}\right] .
\end{aligned}
$$

The initial condition for the model 2 takes the form $N_{1}(\theta)$ $=\psi_{1}(\theta) \geq 0, N_{2}(\theta)=\psi_{2}(\theta) \geq 0, \theta \in\left[-\min \left\{\tau_{1}, \tau_{2}\right\}, 0\right]$, $\psi_{1}(0)>0, \psi_{2}(0)>0,\left(\psi_{1}(\theta), \psi_{2}(\theta)\right) \in\left(C\left([-\tau, 0], \Re_{+}\right)\right.$.

\section{Positivity and boundedness of the delayed system}

In theoretical ecology, positivity and boundedness of a system implies that the system is biologically well behaved. What we mean that for any system, it should have these restrictions (positivity and boundedness) as far as biological aspects are concerned. The following proposition ensures the positivity and boundedness of the delayed system 3.

Proposition 1 Each and every component of the solution of the delayed system 3 is positive and bounded for all $t \geq 0$.

Proof Since the right hand side of the system 3 is completely continuous and locally Lipschitzian on $C$, the solution $\left(N_{1}(t), N_{2}(t)\right)$ of 3 exists and is unique on $[0, \xi)$, where $0<\xi \leqslant \infty$ (Hallam and Luna 1984). From system 3 , we have

$N_{1}(t)=N_{1}(0)\left[\int_{0}^{t}\left(K_{1}-\alpha_{1} N_{1}(s)-\beta_{12} N_{2}(s)-\gamma_{1} N_{1}(s) N_{2}\left(s-\tau_{2}\right)\right) d s\right]>0$,
$N_{2}(t)=N_{2}(0)\left[\int_{0}^{t}\left(K_{2}-\alpha_{2} N_{2}(s)-\beta_{21} N_{1}(s)-\gamma_{2} N_{1}\left(s-\tau_{1}\right) N_{2}(s)\right) d s\right]>0$.

Therefore, $\quad N_{1}(t)>0, N_{2}(t)>0 \quad \forall t \geqslant 0$. Now we assume,

$$
W=N_{1}(t)+N_{2}(t) .
$$

Then,

$$
\begin{gathered}
\frac{d W}{d t} \leqslant-\alpha_{1}\left(N_{1}-\frac{K_{1}}{\alpha_{1}}\right)^{2}-\alpha_{2}\left(N_{2}-\frac{K_{2}}{\alpha_{2}}\right)^{2}+\frac{K_{1}^{2}}{\alpha_{1}} \\
+\frac{K_{2}^{2}}{\alpha_{2}}-K_{1} N_{1}-K_{2} N_{2} \\
\leqslant \mu-v W, \quad \text { where } \mu=\frac{K_{1}^{2}}{\alpha_{1}}+\frac{K_{2}^{2}}{\alpha_{2}} \quad \text { and } \\
v=\min \left\{K_{1}, K_{2}\right\} .
\end{gathered}
$$

Therefore,

$\frac{d W}{d t}+v W \leqslant \mu$.

Applying a theorem on differential inequalities (Birkhoff and Rota 1982), we obtain

$0<W\left(N_{1}, N_{2}\right) \leqslant \frac{\mu}{v}+\frac{W\left(N_{1}(0), N_{2}(0)\right)}{e^{v t}}$

and for $t \rightarrow \infty$,

$0<W \leqslant \frac{\mu}{v}$.

Thus, entire solutions of the system 3 enter into the region $B=\left\{\left(N_{1}, N_{2}\right): 0<W<\frac{\mu}{v}+\varepsilon\right.$ for any $\left.\varepsilon>0\right\}$.

This proves the theorem. 


\section{Imprecise competition model}

All the parameters of the competition model 3 are positive and precise. But due to lack of proper information of the data, the parameters are not always precise. Now if any of the parameters $K_{i}, \alpha_{i}, \beta_{i j}, \gamma_{i}(i \neq j, i, j=1,2)$ is imprecise, i.e., if any parameter is interval number rather than a single value, then it becomes difficult to convert the equation to the standard form and analyze the dynamical behavior of the system. For imprecise parameters, we present the system 3 with interval parameters as described below:

\section{Competition model with interval parameters}

\section{Case I: Toxic inhibition}

Let $\hat{K_{i}}, \hat{\alpha_{i}}, \hat{\beta_{i j}}, \hat{\gamma_{i}}(i \neq j, i, j=1,2)$ be the interval counterparts of $K_{i}, \alpha_{i}, \beta_{i j}, \gamma_{i}$ respectively. Then the imprecise competition delay model 3 becomes

$$
\begin{aligned}
& \frac{d N_{1}}{d t}=N_{1}\left[\hat{K_{1}}-\hat{\alpha_{1}} N_{1}-\hat{\beta_{12}} N_{2}-\hat{\gamma_{1}} N_{1} N_{2}\left(t-\tau_{2}\right)\right], \\
& \frac{d N_{2}}{d t}=N_{2}\left[\hat{K_{2}}-\hat{\alpha_{2}} N_{2}-\hat{\beta_{21}} N_{1}-\hat{\gamma_{2}} N_{1}\left(t-\tau_{1}\right) N_{2}\right],
\end{aligned}
$$

where $\hat{K_{i}}=\left[K_{i l}, K_{i u}\right], \hat{\alpha_{i}}=\left[\alpha_{i l}, \alpha_{i u}\right], \hat{\beta_{i j}}=\left[\beta_{i j l}, \beta_{i j u}\right], \hat{\gamma_{i}}=$ $\left[\gamma_{i l}, \gamma_{i u}\right]$, and $K_{i l}>0, \alpha_{i l}>0, \beta_{i j l}>0, \quad \gamma_{i l}>0 \quad(i \neq j$, $i, j=1,2)$.

\section{Case II: Toxic stimulation}

In this case, $\hat{\gamma_{1}}=-\hat{\gamma}_{1}^{\prime}$ and $\hat{\gamma_{2}}=-\hat{\gamma}_{2}^{\prime}$ where $\hat{\gamma}_{1}^{\prime}=\left[\gamma_{1 l}^{\prime}, \gamma_{1 u}^{\prime}\right]$ and $\hat{\gamma}_{2}^{\prime}=\left[\gamma_{2 l}^{\prime}, \gamma_{2 u}^{\prime}\right]$ and $\hat{\gamma}_{1 l}^{\prime}>0, \hat{\gamma}_{2 l}^{\prime}>0$, i.e., the last term in the Eq. (4) give a positive effect instead of negative effect as in case I. Then the imprecise competition delay model 3 becomes $\frac{d N_{1}}{d t}=N_{1}\left[\hat{K_{1}}-\hat{\alpha_{1}} N_{1}-\hat{\beta_{12}} N_{2}+\hat{\gamma_{1}} N_{1} N_{2}\left(t-\tau_{2}\right)\right]$, $\frac{d N_{2}}{d t}=N_{2}\left[\hat{K_{2}}-\hat{\alpha_{2}} N_{2}-\hat{\beta_{21}} N_{1}+\hat{\gamma_{2}} N_{1}\left(t-\tau_{1}\right) N_{2}\right]$.

\section{Competition model with parametric interval parameters}

For fixed $m$, we are considering the interval-valued function $\phi_{m}(p)=a_{m}^{(1-p)} b_{m}^{p}$ for $p \in[0,1]$ for an interval $\left[a_{m}, b_{m}\right]$. Since $\phi_{m}^{p}$ is a strictly increasing and continuous function, the system 4 and 5 can be written in the parametric form as follows:

$$
\begin{aligned}
\frac{d N_{1}(t ; p)}{d t} & =N_{1}\left[\left(K_{1 l}\right)^{(1-p)}\left(K_{1 u}\right)^{p}-\left(\alpha_{1 l}\right)^{(1-p)}\left(\alpha_{1 u}\right)^{p} N_{1}\right. \\
& \left.-\left(\beta_{12 l}\right)^{(1-p)}\left(\beta_{12 u}\right)^{p} N_{2}-\left(\gamma_{1 l}\right)^{(1-p)}\left(\gamma_{1 u}\right)^{p} N_{1} N_{2}\left(t-\tau_{2}\right)\right], \\
\frac{d N_{2}(t ; p)}{d t} & =N_{2}\left[\left(K_{2 l}\right)^{(1-p)}\left(K_{2 u}\right)^{p}-\left(\alpha_{2 l}\right)^{(1-p)}\left(\alpha_{2 u}\right)^{p} N_{2}\right. \\
& \left.-\left(\beta_{21 l}\right)^{(1-p)}\left(\beta_{21 u}\right)^{p} N_{1}-\left(\gamma_{2 l}\right)^{(1-p)}\left(\gamma_{2 u}\right)^{p} N_{1}\left(t-\tau_{1}\right) N_{2}\right]
\end{aligned}
$$

and

$$
\begin{aligned}
\frac{d N_{1}(t ; p)}{d t} & =N_{1}\left[\left(K_{1 l}\right)^{(1-p)}\left(K_{1 u}\right)^{p}-\left(\alpha_{1 l}\right)^{(1-p)}\left(\alpha_{1 u}\right)^{p} N_{1}\right. \\
& \left.-\left(\beta_{12 l}\right)^{(1-p)}\left(\beta_{12 u}\right)^{p} N_{2}+\left(\gamma_{1 l}\right)^{(1-p)}\left(\gamma_{1 u}\right)^{p} N_{1} N_{2}\left(t-\tau_{2}\right)\right], \\
\frac{d N_{2}(t ; p)}{d t} & =N_{2}\left[\left(K_{2 l}\right)^{(1-p)}\left(K_{2 u}\right)^{p}-\left(\alpha_{2 l}\right)^{(1-p)}\left(\alpha_{2 u}\right)^{p} N_{2}\right. \\
& \left.-\left(\beta_{21 l}\right)^{(1-p)}\left(\beta_{21 u}\right)^{p} N_{1}+\left(\gamma_{2 l}\right)^{(1-p)}\left(\gamma_{2 u}\right)^{p} N_{1}\left(t-\tau_{1}\right) N_{2}\right],
\end{aligned}
$$

where $p \in[0,1]$.

\section{Equilibria and local stability of the delayed system}

\section{Toxic inhibition}

The system 6 has four positive steady states, namely $(i)$ $E_{0}(0,0)$, the trivial equilibrium, (ii) $E_{1}\left(\bar{N}_{1}, 0\right)$ and $E_{2}\left(0, \bar{N}_{2}\right)$, the axial equilibrium, where

$$
\begin{aligned}
& \bar{N}_{1}=\frac{\left(K_{1 l}\right)^{(1-p)}\left(K_{1 u}\right)^{p}}{\left(\alpha_{1 l}\right)^{(1-p)}\left(\alpha_{1 u}\right)^{p}}>0 \text { (always), } \\
& \bar{N}_{2}=\frac{\left(K_{2 l}\right)^{(1-p)}\left(K_{2 u}\right)^{p}}{\left(\alpha_{2 l}\right)^{(1-p)}\left(\alpha_{2 u}\right)^{p}}>0 \text { (always) for all } p \in[0,1]
\end{aligned}
$$

and (iii) $E^{*}=\left(N_{1}^{*}, N_{2}^{*}\right)$, the interior equilibrium, where $N_{1}^{*}, N_{2}^{*}$ can be determined by

$a_{i j} N_{i}^{* 2}+b_{i j} N_{i}^{*}+c_{i j}=0, \quad i \neq j, i, j=1,2$

where

$$
\begin{aligned}
a_{i j}= & \left(\beta_{i j l}\right)^{(1-p)}\left(\beta_{i j u}\right)^{p}\left(\gamma_{i l}\right)^{(1-p)}\left(\gamma_{i u}\right)^{p} \\
& -\left(\alpha_{i l}\right)^{(1-p)}\left(\alpha_{i u}\right)^{p}\left(\gamma_{j l}\right)^{(1-p)}\left(\gamma_{j u}\right)^{p}, \\
b_{i j}= & \left(K_{i l}\right)^{(1-p)}\left(K_{i u}\right)^{p}\left(\gamma_{j l}\right)^{(1-p)}\left(\gamma_{j u}\right)^{p} \\
& -\left(K_{j l}\right)^{(1-p)}\left(K_{j u}\right)^{p}\left(\gamma_{i l}\right)^{(1-p)}\left(\gamma_{i u}\right)^{p} \\
& -\left(\alpha_{i l}\right)^{(1-p)}\left(\alpha_{i u}\right)^{p}\left(\alpha_{j l}\right)^{(1-p)}\left(\alpha_{j u}\right)^{p} \\
& +\left(\beta_{i j l}\right)^{(1-p)}\left(\beta_{i j u}\right)^{p}\left(\beta_{j i l}\right)^{(1-p)}\left(\beta_{j i u}\right)^{p}, \\
c_{i j}= & \left(K_{i l}\right)^{(1-p)}\left(K_{i u}\right)^{p}\left(\alpha_{j l}\right)^{(1-p)}\left(\alpha_{j u}\right)^{p} \\
& -\left(K_{j l}\right)^{(1-p)}\left(K_{j u}\right)^{p}\left(\beta_{j l}\right)^{(1-p)}\left(\beta_{j u}\right)^{p},
\end{aligned}
$$

for all $p \in[0,1]$. Then

$$
N_{i}^{*}=\frac{1}{2 a_{i j}}\left(-b_{i j} \pm \sqrt{b_{i j}^{2}-4 a_{i j} c_{i j}}\right), \quad i, j=1,2
$$

exist with the conditions

$a_{i j} \neq 0, b_{i j}^{2}-4 a_{i j} c_{i j} \geq 0$.

The variational matrix of the system 6 at $E_{0}(0,0)$ is given by 
$V\left(E_{0}\right)=\left[\begin{array}{cc}\left(K_{1 l}\right)^{(1-p)}\left(K_{1 u}\right)^{p} & 0 \\ 0 & \left(K_{2 l}\right)^{(1-p)}\left(K_{2 u}\right)^{p}\end{array}\right] . \quad \quad a_{i j}<0$ i.e., if $\frac{\left(\alpha_{i l}\right)^{(1-p)}\left(\alpha_{i u}\right)^{p}}{\left(\beta_{j i l}\right)^{(1-p)}\left(\beta_{j i u}\right)^{p}}>\frac{\left(\gamma_{i l}\right)^{(1-p)}\left(\gamma_{i u}\right)^{p}}{\left(\gamma_{j l}\right)^{(1-p)}\left(\gamma_{j u}\right)^{p}}$

Clearly, $E_{0}(0,0)$ is a unstable node.

$(i \neq j ; i, j=1,2)$ for all $p \in[0,1]$.

The variational matrix of the system 6 at $E_{1}\left(\bar{N}_{1}, 0\right)$ is given by

So combining Eq. (15) and (16), we have,

$V\left(E_{1}\right)=\left[\begin{array}{cc}-\left(\alpha_{1 l}\right)^{(1-p)}\left(\alpha_{1 u}\right)^{p} \bar{N}_{1} & -\left(\beta_{12 l}\right)^{(1-p)}\left(\beta_{12 u}\right)^{p} \bar{N}_{1}-\left(\gamma_{1 l}\right)^{(1-p)}\left(\gamma_{1 u}\right)^{p} \bar{N}_{1}^{2} e^{-\lambda \tau} \\ 0 & \left(K_{2 l}\right)^{(1-p)}\left(K_{2 u}\right)^{p}-\left(\beta_{21 l}\right)^{(1-p)}\left(\beta_{21 u}\right)^{p} \bar{N}_{1}\end{array}\right]$

then the eigenvalues are $\lambda_{1}=-\left(\alpha_{1 l}\right)^{(1-p)}\left(\alpha_{1 u}\right)^{p}$ $\bar{N}_{1}<0$ (always) and $\lambda_{2}=\left(K_{2 l}\right)^{(1-p)}\left(K_{2 u}\right)^{p}-\left(\beta_{21 l}\right)^{(1-p)}$ $\left(\beta_{21 u}\right)^{p} \bar{N}_{1}$. Therefore the equilibrium point $E_{1}\left(\bar{N}_{1}, 0\right)$ is asymptotically stable if $\frac{\left(K_{1 l}\right)^{(1-p)}\left(K_{1 u}\right)^{p}}{\left(K_{2 l}\right)^{(1-p)}\left(K_{2 u}\right)^{p}}>\frac{\left(\alpha_{11}\right)^{(1-p)}\left(\alpha_{14}\right)^{p}}{\left(\beta_{211}\right)^{(1-p)}\left(\beta_{21 u}\right)^{p}}$ and unstable (saddle point) if

$\frac{\left(K_{1 l}\right)^{(1-p)}\left(K_{1 u}\right)^{p}}{\left(K_{2 l}\right)^{(1-p)}\left(K_{2 u}\right)^{p}}<\frac{\left(\alpha_{1 l}\right)^{(1-p)}\left(\alpha_{1 u}\right)^{p}}{\left(\beta_{21 l}\right)^{(1-p)}\left(\beta_{21 u}\right)^{p}}$.

Again, the variational matrix of the system 6 at $E_{2}\left(0, \bar{N}_{2}\right)$ is given by

$V\left(E_{2}\right)=\left[\begin{array}{cc}\left(K_{1 l}\right)^{(1-p)}\left(K_{1 u}\right)^{p}-\left(\beta_{12 l}\right)^{(1-p)}\left(\beta_{12 u}\right)^{p} \bar{N}_{2} & 0 \\ -\left(\beta_{21 l}\right)^{(1-p)}\left(\beta_{21 u}\right)^{p} \bar{N}_{2}-\left(\gamma_{2 l}\right)^{(1-p)}\left(\gamma_{2 u}\right)^{p} \bar{N}_{2}^{2} e^{-\lambda \tau} & -\left(\alpha_{2 l}\right)^{(1-p)}\left(\alpha_{2 u}\right)^{p} \bar{N}_{2}\end{array}\right]$

then the eigenvalues are $\lambda_{1}=-\left(\alpha_{2 l}\right)^{(1-p)}\left(\alpha_{2 u}\right)^{p}$ $\bar{N}_{2}<0$ (always) and $\lambda_{2}=\left(K_{1 l}\right)^{(1-p)}\left(K_{1 u}\right)^{p}-\left(\beta_{12 l}\right)^{(1-p)}$ $\left(\beta_{12 u}\right)^{p} \bar{N}_{2}$. Therefore the equilibrium point $E_{2}\left(0, \bar{N}_{2}\right)$ is asymptotically stable if $\frac{\left(K_{2 l}\right)^{(1-p)}\left(K_{2 u}\right)^{p}}{\left(K_{1 l}\right)^{(1-p)}\left(K_{1 u}\right)^{p}}>\frac{\left(\alpha_{2 l}\right)^{(1-p)}\left(\alpha_{2 u}\right)^{p}}{\left(\beta_{12 l}\right)^{(1-p)}\left(\beta_{12 u}\right)^{p}}$ and unstable (saddle point) if

$\frac{\left(K_{2 l}\right)^{(1-p)}\left(K_{2 u}\right)^{p}}{\left(K_{1 l}\right)^{(1-p)}\left(K_{1 u}\right)^{p}}<\frac{\left(\alpha_{2 l}\right)^{(1-p)}\left(\alpha_{2 u}\right)^{p}}{\left(\beta_{12 l}\right)^{(1-p)}\left(\beta_{12 u}\right)^{p}}$.

Hence, combining 13 and 14, the condition requires for the persistence of both the species is

$$
\frac{\left(K_{i l}\right)^{(1-p)}\left(K_{i u}\right)^{p}}{\left(K_{j l}\right)^{(1-p)}\left(K_{j u}\right)^{p}}<\frac{\left(\alpha_{i l}\right)^{(1-p)}\left(\alpha_{i u}\right)^{p}}{\left(\beta_{j i l}\right)^{(1-p)}\left(\beta_{j i u}\right)^{p}} \quad(i \neq j ; i, j=1,2)
$$

for all $p \in[0,1]$.

Condition (15) gives $c_{i j}>0$ in Eq. (9). Then the system 6 has unique positive equilibrium if $\frac{\left(\alpha_{i l}\right)^{(1-p)}\left(\alpha_{i u}\right)^{p}}{\left(\beta_{j i l}\right)^{(1-p)}\left(\beta_{j i u}\right)^{p}}>\max \left\{\frac{\left(\gamma_{i l}\right)^{(1-p)}\left(\gamma_{i u}\right)^{p}}{\left(\gamma_{j l}\right)^{(1-p)}\left(\gamma_{j u}\right)^{p}}, \frac{\left(K_{i l}\right)^{(1-p)}\left(K_{i u}\right)^{p}}{\left(K_{j l}\right)^{(1-p)}\left(K_{j u}\right)^{p}}\right\}$

$(i \neq j ; i, j=1,2)$ for all $p \in[0,1]$

as the condition of existence of unique positive interior equilibrium of the system 6 . Now to investigate the local stability of the interior equilibrium $E^{*}\left(N_{1}^{*}, N_{2}^{*}\right)$, we linearize the system 6 by using transformation

$N_{1}=N_{1}^{*}+n_{1}, \quad N_{2}=N_{2}^{*}+n_{2}$.
Linearizing the system 6 at $E^{*}\left(N_{1}^{*}, N_{2}^{*}\right)$ and its corresponding characteristic equation is given by

$$
\begin{aligned}
\lambda^{2} & +A \lambda+B+\left(C_{1} \lambda+D_{1}\right) e^{-\lambda \tau_{1}}+\left(C_{2} \lambda+D_{2}\right) e^{-\lambda \tau_{2}} \\
& +E e^{-\lambda\left(\tau_{1}+\tau_{2}\right)}=0,
\end{aligned}
$$

where $\quad A=-\left(a_{11}+a_{22}\right), \quad D_{1}=a_{22} b_{11}, \quad C_{1}=-b_{11}$, $D_{2}=a_{11} c_{22}, \quad C_{2}=-c_{22}, \quad E=b_{11} c_{22}, \quad B=\left(a_{11} a_{22}-\right.$ $\left.a_{12} a_{21}\right), \quad a_{11}=-\left\{\left(\alpha_{1 l}\right)(1-p)\left(\alpha_{1 u}\right)^{p}+\left(\gamma_{1 l}\right)(1-p)\left(\gamma_{1 u}\right)^{p}\right.$ $\left.N_{2}^{*}\right\} N_{1}^{*}, \quad a_{12}=-\left(\beta_{12 l}\right)(1-p)\left(\beta_{12 u}\right)^{p} N_{1}^{*}, \quad a_{21}=-\left(\beta_{21 l}\right)$ $(1-p)\left(\beta_{21 u}\right)^{p} N_{2}^{*}, \quad a_{22}=-\left\{\left(\alpha_{2 l}\right)(1-p)\left(\alpha_{21 u}\right)^{p}+\left(\gamma_{2 l}\right)\right.$ $\left.\left.(1-p)\left(\gamma_{2 u}\right)^{p} N_{1}^{*}\right\} N_{2}^{*}, \quad b_{11}=\left(\gamma_{2 l}\right)^{(} 1-p\right) \quad\left(\gamma_{2 u}\right)^{p} N_{2}^{* 2}, c_{22}=$

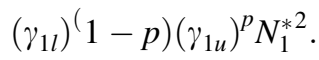

Case $1 \tau_{1}=0=\tau_{2}$

In case of $\tau_{1}=0=\tau_{2}$, the Eq. (18) becomes

$\lambda^{2}+\left(A+C_{1}+C_{2}\right) \lambda+\left(B+D_{1}+D_{2}+E\right)=0$.

All roots of the Eq. (19) have negative real parts if and only if 
$A+C_{1}+C_{2}>0$ and $B+D_{1}+D_{2}+E>0$.

Theorem 2.1 If $\tau_{1}=\tau_{2}=0$, then interior equilibrium point $E^{*}\left(N_{1}^{*}, N_{2}^{*}\right)$ of system (6) exists and asymptotically stable if condition (16) and (20) hold simultaneously.

Case $2 \tau_{1}=0$ and $\tau_{2} \neq 0$

If $\tau_{1}=0$ and $\tau_{2} \neq 0$. In this case, the characteristic Eq. (18) becomes

$\lambda^{2}+\left(A+C_{1}\right) \lambda+B+D_{1}+\left(C_{2} \lambda+D_{2}+E\right) e^{-\lambda \tau_{2}}=0$.

Let $i \omega(\omega>0)$ be a root of the Eq. (21). Then we have,

$\left(D_{2}+E\right) \cos \omega \tau_{2}+C_{2} \omega \sin \omega \tau_{2}=\omega^{2}-\left(B+D_{1}\right)$,

$C_{2} \omega \cos \omega \tau_{2}-\left(D_{2}+E\right) \sin \omega \tau_{2}=-\left(A+C_{1}\right) \omega$.

This leads to

$$
\begin{aligned}
\omega^{4}- & {\left[C_{2}^{2}-\left(A+C_{1}\right)^{2}+2\left(B+D_{1}\right)\right] \omega^{2} } \\
+ & \left(B+D_{1}\right)^{2}-\left(D_{2}+E\right)^{2}=0 .
\end{aligned}
$$

It follows that the Eq. (23) has no positive roots if the following conditions are satisfied:

$$
\begin{aligned}
& \left(A+C_{1}\right)^{2}-C_{2}^{2}-2\left(B+D_{1}\right)>0 \quad \text { and } \\
& \left(B+D_{1}\right)^{2}-\left(D_{2}+E\right)^{2}>0 .
\end{aligned}
$$

Hence, all roots of the Eq. (23) will have negative real parts when $\tau_{2} \in[0, \infty)$ if conditions of the Theorem 2.1 and (24) are satisfied. Let

$$
\left(B+D_{1}\right)^{2}-\left(D_{2}+E\right)^{2}<0 .
$$

If Theorems 2.1 and (25) hold then the Eq. (23) has a unique positive root $\omega_{0}^{2}$. Substituting $\omega_{0}^{2}$ into Eq. (22), we have

$$
\begin{aligned}
\tau_{2_{n}}= & \frac{1}{\omega_{0}} \cos ^{-1}\left[\frac{\left(D_{2}+E\right)\left(\omega_{0}^{2}-B-D_{1}\right)-\left(A+C_{1}\right) C_{2} \omega_{0}^{2}}{C_{2}^{2} \omega_{0}^{2}+\left(D_{2}+E\right)^{2}}\right] \\
& +\frac{2 n \pi}{\omega_{0}}, \quad n=0,1,2, \ldots
\end{aligned}
$$

Let,

$$
\begin{aligned}
& C_{2}^{2}-\left(A+C_{1}\right)^{2}+2\left(B+D_{1}\right)>0, \\
& \left(B+D_{1}\right)^{2}-\left(D_{2}+E\right)^{2}>0
\end{aligned}
$$

and

$$
\left[C_{2}^{2}-\left(A+C_{1}\right)^{2}+2\left(B+D_{1}\right)\right]^{2}>4\left[\left(B+D_{1}\right)^{2}-\left(D_{2}+E\right)^{2}\right] .
$$

If Theorem 2.1 and (26) hold then Eq. (23) has two positive roots $\omega_{+}^{2}$ and $\omega_{-}^{2}$. Substituting $\omega_{ \pm}$into Eq. (22), we get

$$
\begin{aligned}
\tau_{2_{k}}^{ \pm}= & \frac{1}{\omega_{ \pm}} \cos ^{-1}\left[\frac{\left(D_{2}+E\right)\left(\omega_{ \pm}^{2}-B-D_{1}\right)-\left(A+C_{1}\right) C_{2} \omega_{ \pm}^{2}}{C_{2}^{2} \omega_{ \pm}^{2}+\left(D_{2}+E\right)^{2}}\right] \\
& +\frac{2 k \pi}{\omega_{ \pm}}, \quad k=0,1,2, \ldots
\end{aligned}
$$

If $\lambda\left(\tau_{2}\right)$ be the root of Eq. (21) satisfying $\operatorname{Re} \lambda\left(\tau_{2_{n}}\right)=0$ (respectively, $\operatorname{Re} \lambda\left(\tau_{2_{k}}^{ \pm}\right)=0$ ) and $\operatorname{Im} \lambda\left(\tau_{2_{n}}\right)=\omega_{0}$ (respectively, $\left.\operatorname{Im} \lambda\left(\tau_{2_{k}}^{ \pm}\right)=\omega_{ \pm}\right)$, we get

$$
\begin{aligned}
{\left[\frac{d}{d \tau_{2}} \operatorname{Re}(\lambda)\right]_{\tau_{2}=\tau_{2}, \omega=\omega_{0}} } & =\frac{\omega^{4}+\left(D_{2}+E\right)^{2}-\left(B+D_{1}\right)^{2}}{\omega^{2}\left(C_{2}^{2} \omega^{2}+\left(D_{2}+E\right)^{2}\right)} \\
& >\frac{\omega^{2}}{C_{2}^{2} \omega^{2}+\left(D_{2}+E\right)^{2}}>0(\text { by }(25)) .
\end{aligned}
$$

Similarly, we can show that

$$
\begin{aligned}
& {\left[\frac{d}{d \tau_{2}} \operatorname{Re}(\lambda)\right]_{\tau_{2}=\tau_{2_{k}}^{+}, \omega=\omega_{+}}>0,} \\
& {\left[\frac{d}{d \tau_{2}} \operatorname{Re}(\lambda)\right]_{\tau_{2}=\tau_{2_{k}}^{-}, \omega=\omega_{-}}<0 .}
\end{aligned}
$$

From Corollary (2.4) in Ruan and Wei (2003), we have the following conclusions.

Theorem 2.2 Assume $\tau_{1}=0, \tau_{2} \neq 0$ and conditions of the Theorem 2.1 are satisfied, then the following conclusions hold:

(i) If (24) holds then the equilibrium $E^{*}\left(N_{1}^{*}, N_{2}^{*}\right)$ is asymptotically stable for all $\tau_{2} \geq 0$.

(ii) If (25) holds then the equilibrium $E^{*}\left(N_{1}^{*}, N_{2}^{*}\right)$ is conditionally stable. It is locally asymptotically stable for $\tau_{2}<\tau_{2_{0}}$ and unstable for $\tau_{2}>\tau_{2_{0}}$. Furthermore, the system (6) undergoes a Hopf bifurcation at $E^{*}\left(N_{1}^{*}, N_{2}^{*}\right)$ when $\tau_{2}=\tau_{2_{0}}$, where

$$
\tau_{2_{0}}=\frac{1}{\omega_{0}} \cos ^{-1}\left[\frac{\left(D_{2}+E\right)\left(\omega_{0}^{2}-B-D_{1}\right)-\left(A+C_{1}\right) C_{2} \omega_{0}^{2}}{C_{2}^{2} \omega_{0}^{2}+\left(D_{2}+E\right)^{2}}\right] .
$$

(iii) If (26) holds then there is a positive integer $m$ such that the equilibrium is stable when $\tau_{2} \in$ $\left[0, \tau_{2_{0}}^{+}\right) \cup\left(\tau_{2_{0}}^{-}, \tau_{2_{1}}^{+}\right) \cup \ldots \cup\left(\tau_{2_{m-1}}^{-}, \tau_{2_{m}}^{+}\right)$and unstable when $\tau_{2} \in\left[\tau_{2_{0}}^{+}, \tau_{2_{0}}^{-}\right) \cup\left(\tau_{2_{1}}^{+}, \tau_{2_{1}}^{-}\right) \cup \ldots \cup\left(\tau_{2_{m-1}}^{+}\right.$, $\left.\tau_{2_{m-1}}^{-}\right) \cup\left(\tau_{2_{m}}^{+}, \infty\right)$. Furthermore, the system undergoes a Hopf bifurcation at $E^{*}\left(N_{1}^{*}, N_{2}^{*}\right)$ when $\tau_{2}=\tau_{2_{m}}^{+}, m=0,1,2, \ldots$

Case $3 \tau_{1} \neq 0$ and $\tau_{2} \neq 0$

We consider Eq. (18) with $\tau_{2}$ in its stable interval $\left(\tau_{2} \in\right.$ $\left.\left[0, \tau_{2_{0}}\right)\right)$ and regard $\tau_{1}$ as a parameter $\left(\tau_{1} \in(0, \infty)\right)$. Without loss of generality, we consider that the system 
parameters satisfy Theorem 2.1 and (25). Let $i \omega(\omega>0)$ be a root of Eq. (18) and we obtain

$$
\begin{aligned}
\omega^{4} & +\widetilde{A} \omega^{2}+B^{2}+D_{2}^{2}-D_{1}^{2}-E^{2} \\
& +2 \widetilde{B} \sin \omega \tau_{2}+2 \widetilde{C} \cos \omega \tau_{2}=0
\end{aligned}
$$

where

$$
\begin{aligned}
& \widetilde{A}=A^{2}+C_{2}^{2}-2 B-C_{1}^{2}, \\
& \widetilde{B}=\omega C_{1} E-\omega^{3} C_{2}-\omega A D_{2}+\omega B C_{2} \text { and } \\
& \widetilde{C}=-D_{1} E-\omega^{2} D_{2}+B D_{2}+\omega^{2} A C_{2} .
\end{aligned}
$$

We define,

$$
\begin{aligned}
F(\omega)= & \omega^{4}+\widetilde{A} \omega^{2}+B^{2}+D_{2}^{2}-D_{1}^{2} \\
& -E^{2}+2 \widetilde{B} \sin \omega \tau_{2}+2 \widetilde{C} \cos \omega \tau_{2}
\end{aligned}
$$

and assume that

$$
\left(B+D_{2}\right)^{2}-\left(D_{1}+E\right)^{2}<0 .
$$

Then it is easy to check that $F(0)<0$ and $F(\infty)=\infty$. Thus, (27) has finite positive roots $\omega_{1}, \omega_{2}, \ldots, \omega_{k}$. For every fixed $\omega_{i}, i=1,2, \ldots, k$, there exists a sequence $\left\{\tau_{1_{i}}^{j} \mid j=1,2, \ldots\right\}$, where,

$$
\begin{array}{r}
\tau_{1_{i}}^{j}=\left(\frac{1}{\omega_{i}}\right) \cos ^{-1}\left[\frac{M_{1}}{N_{1}}\right]+\frac{2 i \pi}{\omega_{i}}, \\
i=1,2, \ldots, k ; \quad j=1,2, \ldots
\end{array}
$$

where,

$$
\begin{aligned}
M_{1}= & P_{1} S_{1}+P_{2} T_{1}+\left(Q_{1} S_{1}+R_{1} T_{1}\right) \cos \omega_{i} \tau_{2} \\
& +\left(R_{1} S_{1}-Q_{1} T_{1}\right) \sin \omega_{i} \tau_{2}, \\
N_{1}= & S_{1}^{2}+T_{1}^{2}, \quad P_{1}=-\omega_{i}^{2}+B, \quad P_{2}=A \omega_{i}, \\
& Q_{1}=D_{2}, \quad R_{1}=C_{2} \omega_{i}, \\
S_{1}= & -\left(E \cos \omega_{i} \tau_{2}+D_{1}\right), \quad T_{1}=E \sin \omega_{i} \tau_{2}-C_{1} \omega_{i}, \\
& i=1,2, \ldots, k
\end{aligned}
$$

Let $\tau_{1_{0}}=\min \left\{\tau_{1_{i}}^{j} \mid i=1,2, \ldots, k ; j=1,2, \ldots\right\}$. Define the function $\theta\left(\tau_{1}\right) \in[0,2 \pi)$ such that $\cos \theta\left(\tau_{1}\right)$ is given by the right-hand sides of the last equation. In the following, we assume that

$$
\left[\frac{d}{d \tau_{1}}(\operatorname{Re} \lambda)\right]_{\lambda=i \omega_{0}} \neq 0 .
$$

Therefore, by the general Hopf bifurcation theorem, we have the following result on the stability and bifurcation of the system (6).

Theorem 2.3 For the system (6), suppose the parameters satisfy the conditions of the Theorem 2.1, 25 and 29 and $\tau_{2} \in\left[0, \tau_{2_{0}}\right)$, then the equilibrium $E^{*}\left(N_{1}^{*}, N_{2}^{*}\right)$ is asymptotically stable when $\tau_{1} \in\left(0, \tau_{1_{0}}\right)$, unstable when $\tau_{1}>\tau_{1_{0}}$ and a Hopf bifurcation occurs when $\tau_{1}=\tau_{1_{0}}$.
Table 1 Stable equilibrium points of toxic inhibited system for different $p$

\begin{tabular}{lll}
\hline$p$ & Equilibrium points & Fig. 1 \\
\hline 0.0 & $(31.5810,4.8369)$ & $\mathrm{a}$ \\
0.3 & $(26.8610,4.1779)$ & $\mathrm{b}$ \\
0.5 & $(24.1373,3.7626)$ & $\mathrm{c}$ \\
0.8 & $(20.6083,3.1699)$ & $\mathrm{d}$ \\
1.0 & $(18.5878,2.7915)$ & $\mathrm{e}$ \\
\hline
\end{tabular}

Case $4 \tau_{1} \neq 0$ but $\tau_{2}=0$

Next, we assume that $\tau_{1} \neq 0$ but $\tau_{2}=0$. The proof is similar to the Case 2 and we only summarize the results below.

Theorem 2.4 Assume Theorem 2.1 is satisfied, then the following conclusions hold:

(i) If

$$
\begin{aligned}
& \left(A+C_{2}\right)^{2}-C_{1}^{2}-2\left(B+D_{2}\right)>0, \\
& \left.B+D_{2}\right)^{2}-\left(D_{1}+E\right)^{2}>0
\end{aligned}
$$

hold, then the equilibrium $E^{*}\left(N_{1}^{*}, N_{2}^{*}\right)$ is locally asymptotically stable for all $\tau_{1} \geq 0$.

(ii) If

$$
\left(B+D_{2}\right)^{2}-\left(D_{1}+E\right)^{2}<0
$$

holds, then the equilibrium $E^{*}\left(N_{1}^{*}, N_{2}^{*}\right)$ is locally asymptotically stable for $\tau_{1}<\tilde{\tau}_{1_{0}}$ and unstable for $\tau_{1}>\tilde{\tau}_{1_{0}}$. Furthermore, the system (6) undergoes a Hopf bifurcation at $E^{*}\left(N_{1}^{*}, N_{2}^{*}\right)$ when $\tau_{1}=\tilde{\tau}_{1_{0}}$, where

$$
\begin{aligned}
\tilde{\tau}_{1_{p}}= & \frac{1}{\bar{\omega}_{0}} \cos ^{-1}\left[\frac{\left(D_{1}+E\right)\left(\bar{\omega}_{0}^{2}-B-D_{2}\right)-\left(A+C_{2}\right) C_{1} \bar{\omega}_{0}^{2}}{C_{1}^{2} \bar{\omega}_{0}^{2}+\left(D_{1}+E\right)^{2}}\right] \\
& +\frac{2 p \pi}{\bar{\omega}_{0}}, \quad p=0,1,2, \ldots
\end{aligned}
$$

(iii) If $C_{1}^{2}-\left(A+C_{2}\right)^{2}+2\left(B+D_{2}\right)>0,\left(B+D_{2}\right)^{2}-$ $\left(D_{1}+E\right)^{2}>0$ and

$$
\left[C_{1}^{2}-\left(A+C_{2}\right)^{2}+2\left(B+D_{2}\right]^{2}>4\left[\left(B+D_{2}\right)^{2}-\left(D_{1}+E\right)^{2}\right]\right.
$$

hold, there is a positive integer $l$ such that the equilibrium is stable when $\tau_{1} \in\left[0, \tilde{\tau}_{1_{0}}^{+}\right) \cup\left(\tilde{\tau}_{1_{0}}^{-}, \tilde{\tau}_{1_{1}}^{+}\right) \cup \ldots \cup$ $\left(\tilde{\tau}_{1_{l-1}}^{-}, \tilde{\tau}_{1_{l}}^{+}\right)$, and unstable when $\tau_{1} \in\left[\tilde{\tau}_{1_{0}}^{+}, \tilde{\tau}_{1_{0}}^{-}\right) \cup\left(\tilde{\tau}_{1_{1}}^{+}, \tilde{\tau}_{1_{1}}^{-}\right) \cup$ $\ldots \cup\left(\tilde{\tau}_{1_{l-1}}^{+}, \tilde{\tau}_{1_{l-1}}^{-}\right) \cup\left(\tilde{\tau}_{1_{l}}^{+}, \infty\right)$. Furthermore, the system undergoes a Hopf bifurcation at $\left(N_{1}^{*}, N_{2}^{*}\right)$ when $\tau_{1}=\tilde{\tau}_{1_{l}}^{+}, \quad l=0,1,2, \ldots$

Case $5 \tau_{1} \neq 0$ and $\tau_{2} \neq 0$ 

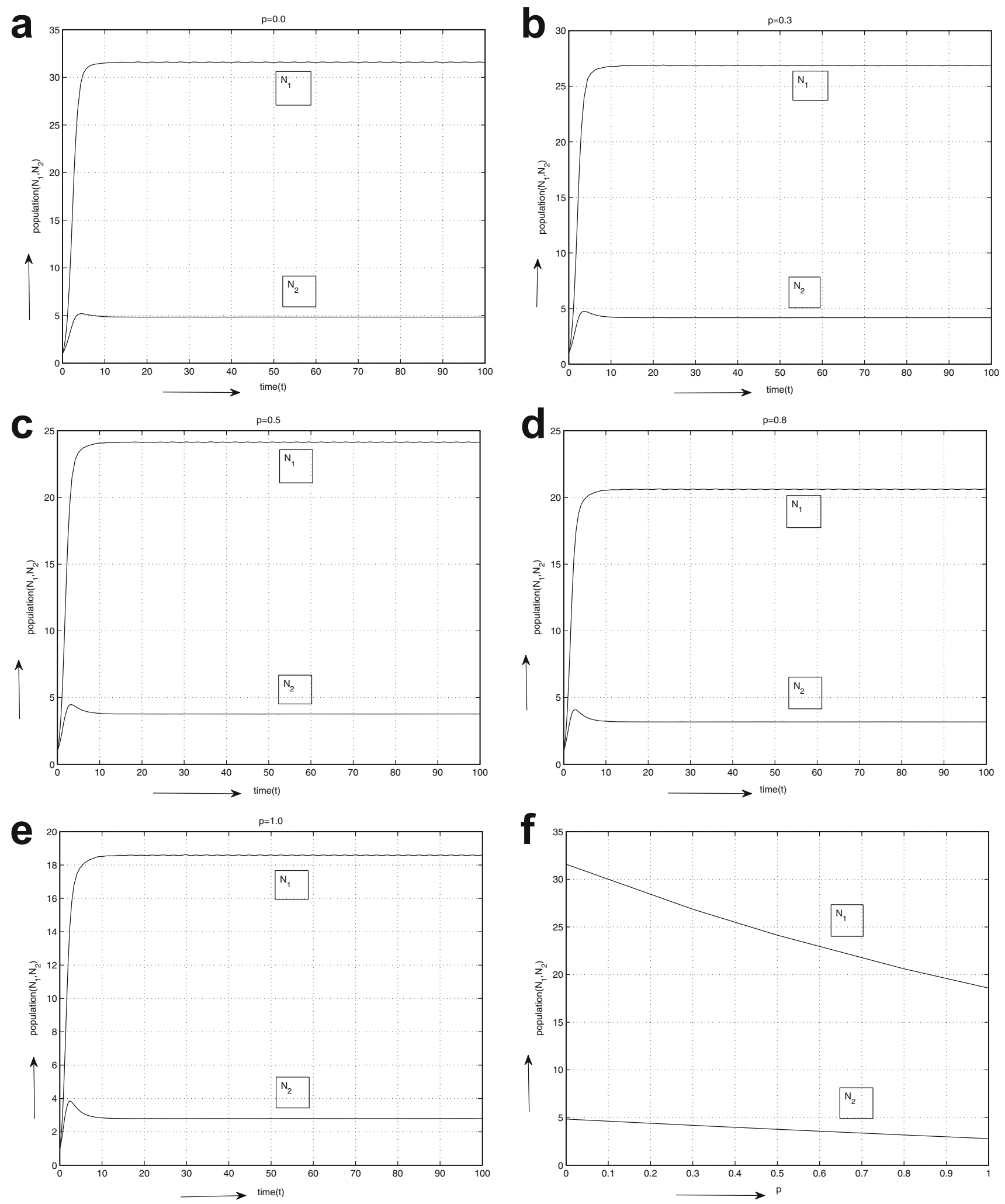

Fig. 1 Behavior of the toxic inhibited species with time by using the imprecise parameters values and initial conditions $\left(N_{1}(0), N_{2}(0)\right)=$ $(1.0,1.0)$ for $p=0.0,0.3,0.5,0.8,1.0$ and Fig. 1f shows the

dynamical behavior of the two species population $\left(N_{1}, N_{2}\right)$ with respect to $p$ when the values of the other parameters are same 
Table 2 Stable equilibrium points of toxic stimulated system for different $p$

\begin{tabular}{lll}
\hline$p$ & Equilibrium points & Fig. 2 \\
\hline 0.0 & $(16.9842,41.4162)$ & $\mathrm{a}$ \\
0.3 & $(13.4222,33.1089)$ & $\mathrm{b}$ \\
0.5 & $(11.4207,28.6961)$ & $\mathrm{c}$ \\
0.8 & $(8.8834,23.3342)$ & $\mathrm{d}$ \\
1.0 & $(7.4548,20.4188)$ & $\mathrm{e}$ \\
\hline
\end{tabular}

Finally, we assume that both the prey and predator are harvested selectively then $\tau_{1} \neq 0$ and $\tau_{2} \neq 0$. We consider Eq. (18) with $\tau_{1}$ in its stable interval and regard $\tau_{2}$ as a parameter. The proof is similar to the Case 3 and we only summarize the results below.

Theorem 2.5 For the system (6), suppose the parameters satisfy the conditions of the Theorem 2.1, (25) and (29) and $\tau_{1} \in\left[0, \tau_{1_{0}}\right)$. Then the equilibrium $E^{*}\left(N_{1}^{*}, N_{2}^{*}\right)$ is locally asymptotically stable when $\tau_{2} \in\left(0, \bar{\tau}_{2_{\mathrm{s}}}^{t}\right)$, unstable when $\tau_{2}>\bar{\tau}_{2_{s}}^{t}$ and a Hopf bifurcation occurs when $\tau_{2}=\bar{\tau}_{2_{s}}^{t}$, where,

$$
\begin{gathered}
\bar{\tau}_{2_{s}}^{t}=\left(\frac{1}{\overline{\omega_{s}}}\right) \cos ^{-1}\left[\frac{M_{2}}{N_{2}}\right]+\frac{2 s \pi}{\overline{\omega_{s}}}, \\
s=1,2, \ldots, q ; \quad t=1,2, \ldots
\end{gathered}
$$

where,

$$
\begin{aligned}
M_{2}= & P_{1} S_{2}+P_{2} T_{2}+\left(Q_{2} S_{2}+R_{2} T_{2}\right) \cos \omega_{s} \tau_{1} \\
& +\left(R_{2} S_{2}-Q_{2} T_{2}\right) \sin \omega_{s} \tau_{1}, \\
N_{2}= & S_{2}^{2}+T_{2}^{2}, \quad P_{1}=-\omega_{s}^{2}+B, \quad P_{2}=A \omega_{s}, \\
& d Q_{2}=D_{1}, \quad R_{2}=C_{1} \omega_{s}, \\
S_{2}= & -\left(E \cos \omega_{s} \tau_{1}+D_{2}\right), \quad T_{2}=E \sin \omega_{s} \tau_{1}-C_{2} \omega_{s}, \\
& s=1,2, \ldots, q .
\end{aligned}
$$

\section{Toxic stimulation}

In this case, we study the system (7), which is same as the system (6) unless in the last term, where the toxic inhibition rate $\gamma_{i}(i=1,2)$ are negative i.e., either species produces auxin which stimulate the growth of the other species. Here the local stability analysis of the system (7) remains same as in case I for the trivial and axial equilibrium points and so as the criteria for persistence of both the species. Now we study the local stability of the system (7) for the interior equilibrium point $E \digamma^{*}\left(N_{1}^{\prime *}, N_{2}^{\prime *}\right)$ which is also asymptotically stable.
Table 3 Critical values of time delay $\left(\tau^{*}\right)$ and transversality condition (TC) of toxic 6 system for different $p$

\begin{tabular}{llllll}
\hline$p$ & 0.0 & 0.3 & 0.5 & 0.8 & 1.0 \\
\hline$\tau^{*}$ & 0.9294 & 0.9788 & 1.0101 & 1.0585 & 1.0944 \\
TC & $0.5 \mathrm{E}-04$ & 0.0003 & 0.1892 & 0.7332 & 1.0668 \\
\hline
\end{tabular}

\section{Numerical Illustration}

Analytical studies can never be completed without numerical verification of the results. In this section we present computer simulation of some solutions of the system (6) and (7). Beside verification of our analytical findings, these numerical solutions are very important from practical point of view. Let us consider a set of imprecise biological values of parameters as follows in appropriate units: $\hat{K}_{1}=$ $[1.5,2.0], \hat{K_{2}}=[0.8,1.2], \hat{\alpha_{1}}=[0.04,0.09], \hat{\alpha_{2}}=[0.05$, $0.1], \quad \hat{\beta_{12}}=[0.03,0.08], \quad \hat{\beta_{21}}=[0.008,0.03], \hat{\gamma_{1}}=$ $[0.0006,0.002], \hat{\gamma_{2}}=[0.002,0.007]$ and $p \in[0,1]$. Also for the purpose of the execution of delayed system (6), we take a particular example when $\tau_{1}=\tau_{2}=\tau$ (say), then we get the following numerical results:

\section{Case I: Toxic inhibition}

Using the parametric form of interval numbers and assuming the initial condition $\left(N_{1}(0), N_{2}(0)\right)=(1.0,1.0)$, we find the conditions given in (19) are satisfied, which imply that the unique interior equilibrium point $E^{*}\left(N_{1}^{*}, N_{2}^{*}\right)$ exist of the system (6) for all values of $p \in[0,1]$. From Table 1 we observe that for different values of the parameter $p$, the system (6) corresponds a unique positive equilibrium point which are locally asymptotically stable. We present the dynamics of the model for different values of $p(p=0.0,0.3,0.5,0.8,1.0)$ in Fig. 1a-e. These figures show that the interior equilibrium $E^{*}$ exist and asymptotically stable for all values of $p \in[0,1]$. But the values are different for different values of $p$. Figure if shows that both the species population decrease with increasing $p$, but $N_{1}$ species is decreasing rapidly where as $\mathrm{N}_{2}$ species decreasing slowly. However, in this case, analytically we have already shown that the system is always stable for all $\tau \geq 0$.

\section{Case II: Toxic stimulation}

Here also we consider the same parametric form of interval numbers and assuming the initial condition $\left(N_{1}^{\prime}(0), N_{2}^{\prime}(0)\right)=(1.0,1.0)$, we find the conditions given in (29) are satisfied which imply that the unique interior equilibrium point $E^{\prime *}\left(N_{1}^{\prime *}, N_{2}^{\prime *}\right)$ exist of the system (7) for all values of $p \in[0,1]$. Also when the delay value $\tau=0$, the condition (33) is satisfied and the interior equilibrium point $E^{\prime *}$ is locally asymptotically stable. From Table 2, we 

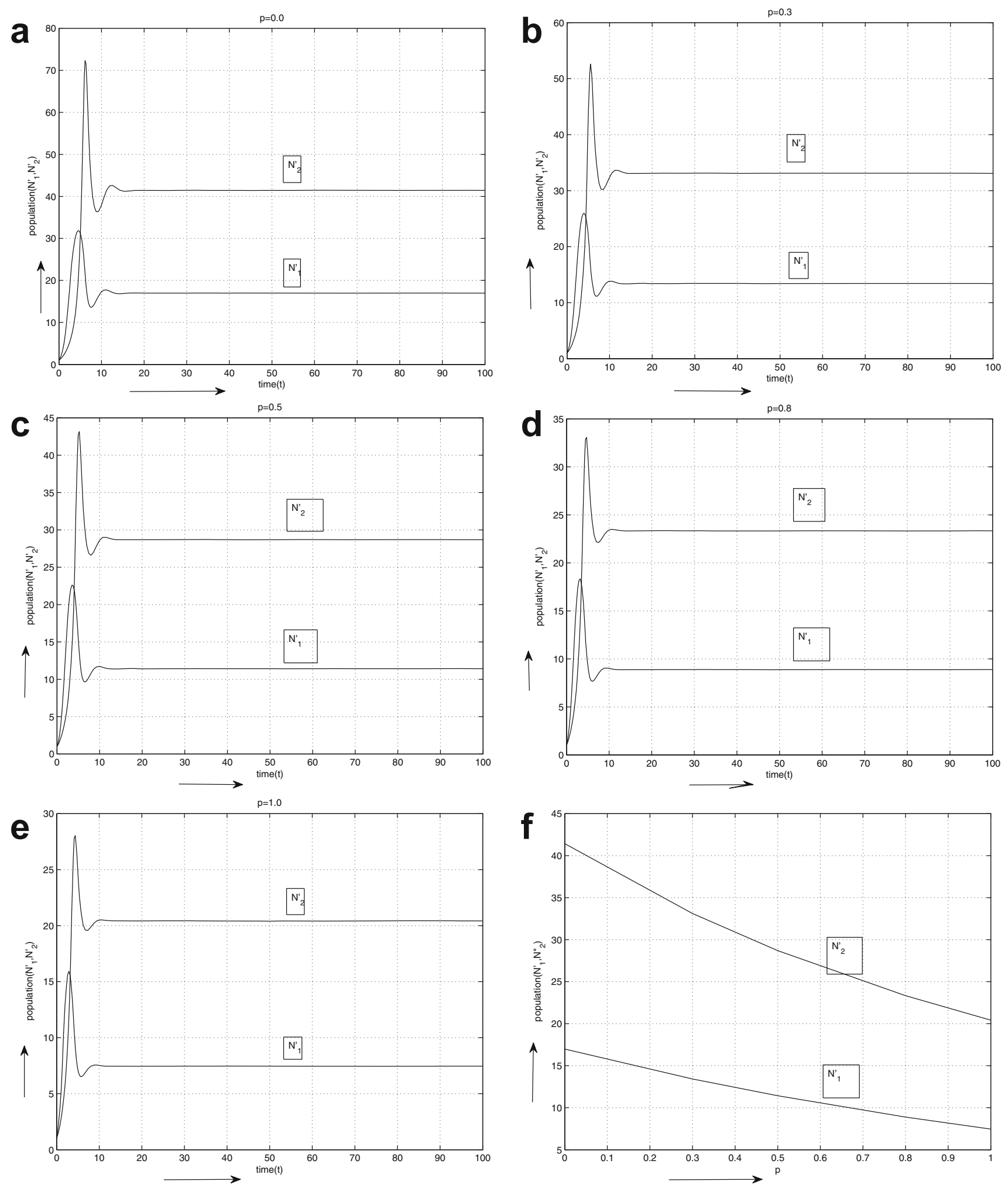

Fig. 2 Behavior of the toxic inhibited species with time by using the imprecise parameters values and initial conditions $\left(N_{1}^{\prime}(0), N_{2}^{\prime}(0)\right)=$ dynamical behavior of the two species population $\left(N_{1}^{\prime}, N_{2}^{\prime}\right)$ with respect to $p$ when the values of the other parameters are same 

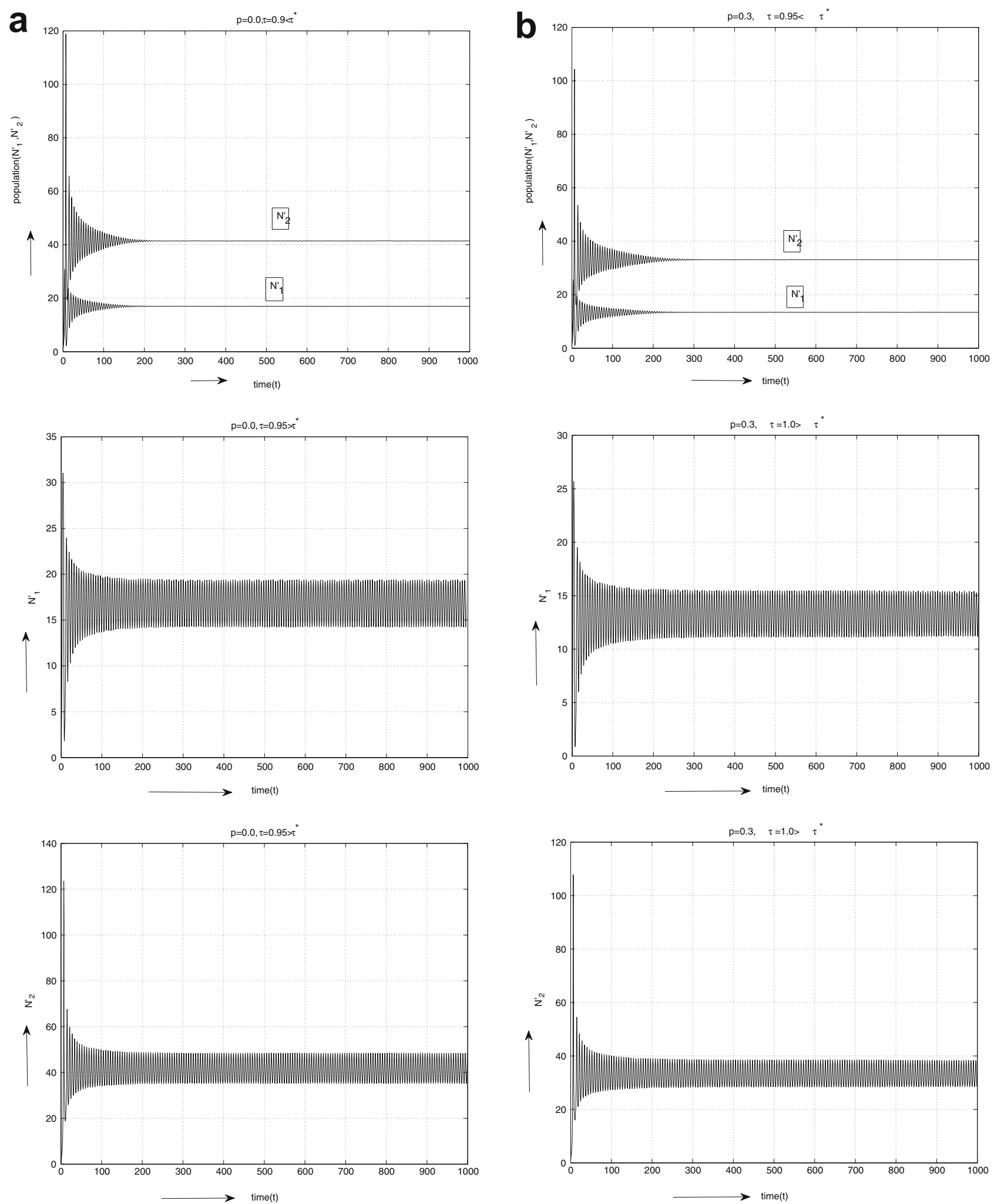

Fig. 3 Time course of two competitive species variation when $\tau<\tau^{*}$ and $\tau>\tau^{*}$ with same imprecise parameter values for $p=0.0,0.3,0.5,0.8,1.0$ 

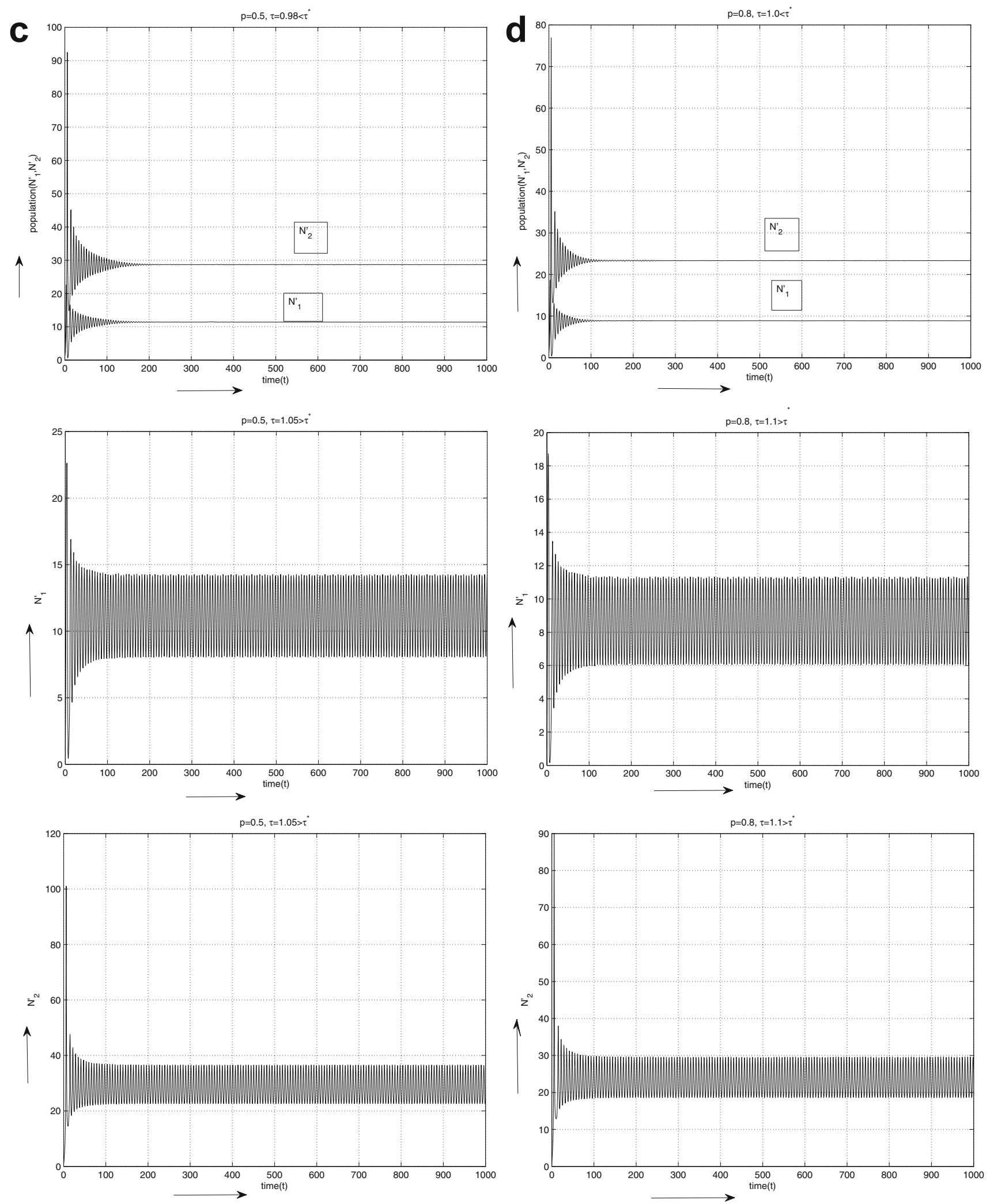

Fig. 3 continued 

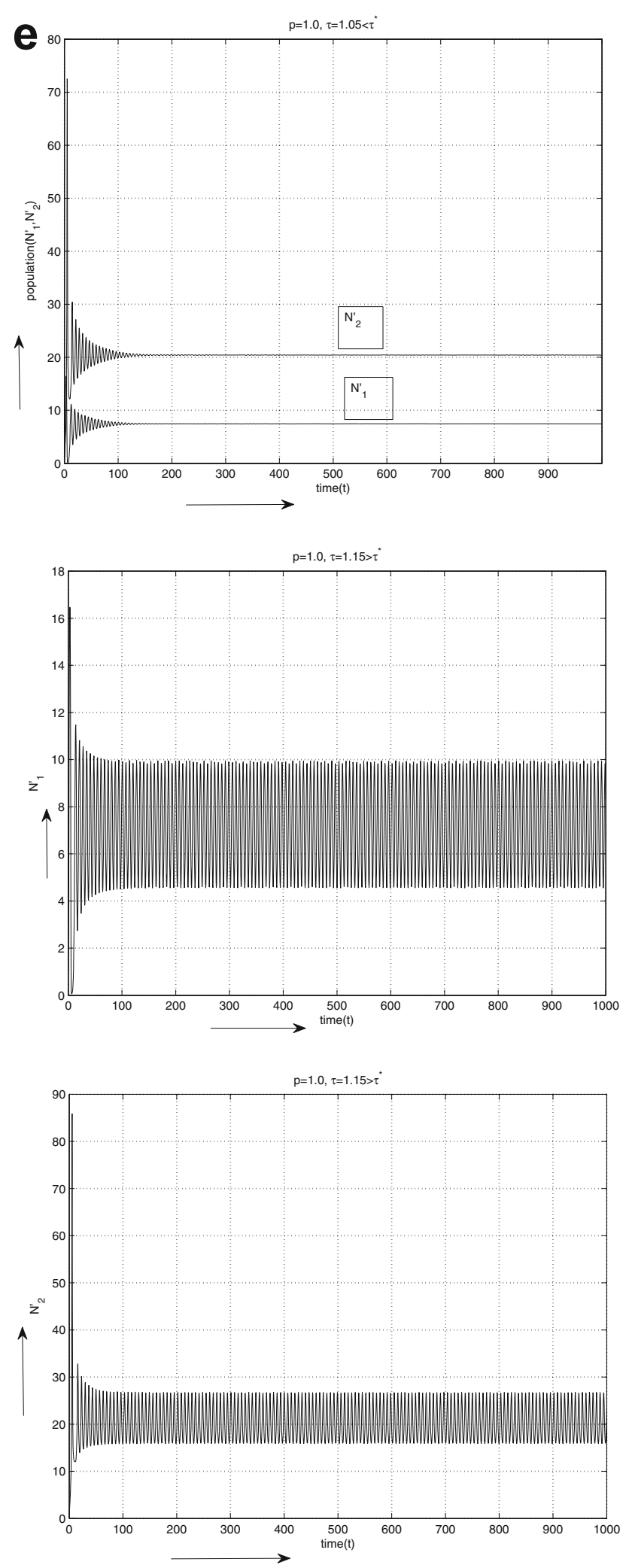

Fig. 3 continued observe that for different values of the parameter $p$, the system (7) corresponds a unique positive equilibrium point which are locally asymptotically stable. we present the dynamics of the model for different values of $p(p=$ $0.0,0.3,0.5,0.8,1.0)$ in Fig. $2 \mathrm{a}-\mathrm{e}$. These figures show that the interior equilibrium $E^{*}$ exist and asymptotically stable for all values of $p \in[0,1]$. But values are different for different values of $p$. Figure $2 \mathrm{f}$ shows that both the species population decrease at more or less same rate with increasing $p$.

Finally we investigate the dynamical behavior of the system (7) numerically in the presence of time delay using the same data in Table 3. Now depending on these values we obtain the critical values of the bifurcation parameter (time delay) $\tau$ say $\tau^{*}$ given in Table 3. For a particular value of $p$ we get a definite critical value of the delay $\tau^{*}$ as shown in Table 3. Now for a particular value of $p$, if value of $\tau$ is below the critical value $\tau^{*}$ then Fig. 3a-e shows that the interior equilibrium point $E^{\prime *}\left(N_{1}^{\prime *}, N_{2}^{\prime *}\right)$ is asymptotically stable and both the species converge to their steady states in finite time. Now if we gradually increase only the value of delay, the stability of the equilibrium point $E^{\prime *}\left(N_{1}^{\prime *}, N_{2}^{\prime *}\right)$ may switch over to periodic oscillation. As $\tau$ passes through its critical value $\tau^{*}$ as per Table $3, E^{*}$ losses its stability as shown in Fig. 3a-e, which is the case of Hopf-bifurcation. Also from Fig. 4a-e, the positive equilibrium point $E^{* *}\left(N_{1}^{\prime *}, N_{2}^{\prime *}\right)$ is unstable with a periodic orbit near $E^{\prime *}\left(N_{1}^{\prime *}, N_{2}^{\prime *}\right)$ as $\tau$ passes through $\tau^{*}$.

\section{Conclusion}

In this paper, we have extensively studied a delayed two species competition model with the effect of toxic substances. The model is introduced from the modified LotkaVolterra type competition of two species which are affected by toxicant. These toxic substances may be toxic inhibitory or stimulatory to the other species, which are discussed comprehensively in our paper. Competition models are mostly based on the assumption that the biological parameters are precisely known. But in reality, it is very difficult to estimate the values of all biological parameters precisely. In this paper, we consider a two species competition model with delay in both species using some imprecise parameters. Here we introduce the concept of interval numbers to the model by considering the biological parameters $\hat{K_{1}}, \hat{K_{2}}, \hat{\alpha_{1}}, \hat{\alpha_{2}}, \hat{\beta_{12}}, \hat{\beta_{21}}, \hat{\gamma_{1}}, \hat{\gamma_{2}}$ which are imprecise in nature. We have used parametric functional form of the interval number to convert the imprecise competition model to the corresponding parametric competition delay model. 

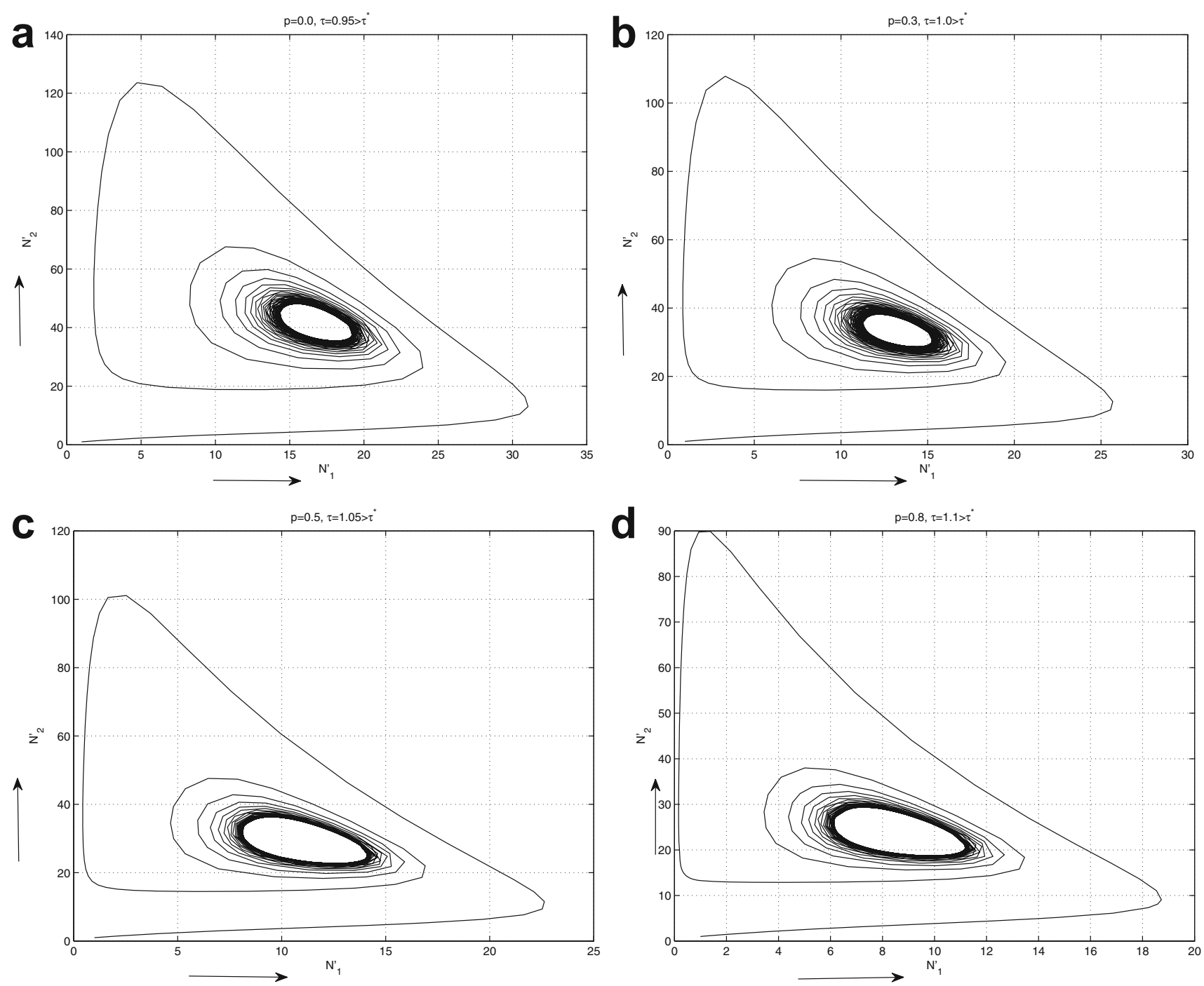

d
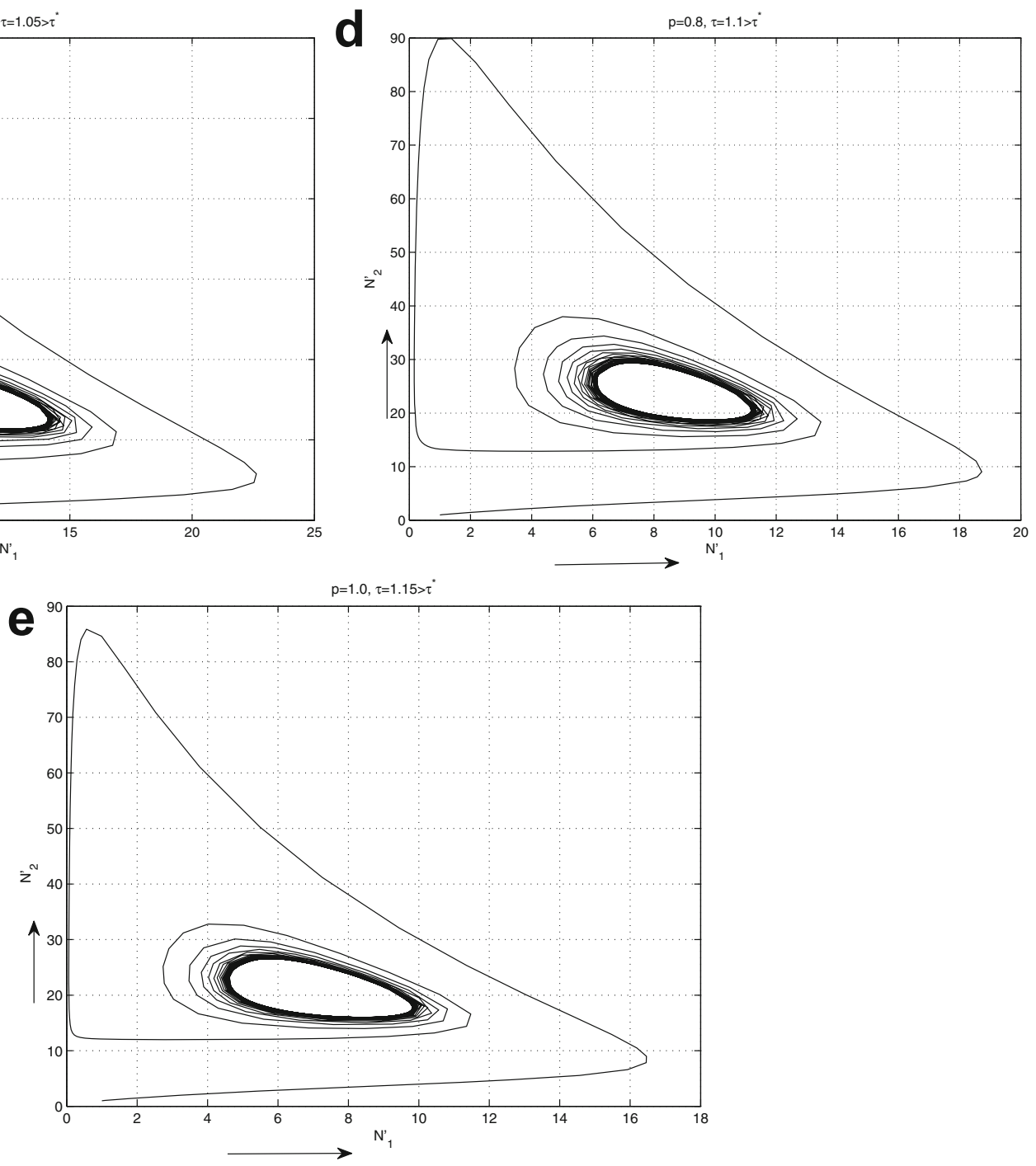

Fig. 4 Phase portrait of species when $\tau>\tau^{*}$ with same imprecise parameter values for $p=0.0,0.3,0.5,0.8,1.0$ 
Dynamical behavior of both the cases, toxic inhibitory and stimulatory model system are examined rigorously in absence as well as presence of time delay for different values of the parameter $p \in[0,1]$. We have discussed the existence and stability of various equilibrium points of both the systems. We obtain different equilibrium points of the species and the critical value of the time delay depending upon values of the parameter $p$. Analytically it is observed that the time delay does not affected the stability on the toxic inhibited system. But in case of toxic stimulatory system, it is observed that the system becomes unstable at different time delay for different value of the parameter $p$ and leads to stable limit cycle periodic solutions through Hopf-bifurcation.

All our important mathematical findings for the dynamical behavior of the two species competition model affected by toxic substances are also numerically verified and graphical representation of a variety of solutions of system (6) and (7) are depicted by using MATLAB with some imprecise parameter values. These numerical results are very important to understand the system in both mathematical and ecological point of view. Finally, we conclude that impreciseness of biological parameters has great impact on the behavior of the delay model. We use the concept of interval number to present imprecise competition modeling, which makes the system more realistic as always it is difficult to know the parameter values precisely. Here we consider all the biological parameters are imprecise, except the delay parameters. The delay model can be made more realistic when incorporated with impreciseness in the delay terms, it makes the model more interesting and is left for future work.

\section{References}

Anderson DM (1989) Toxic algae blooms and red tides : a global perspective. Environmental science and toxicology. Elsevier, New York

Bassanezi RC, Barros LC, Tonelli A (2000) Attractors and asymptotic stability for fuzzy dynamical systems. Fuzzy Sets Syst 113:473-483

Berglund H (1969) Stimulation of growth of two marine algae by organic substances excreted by Enteromorpha linza in unialgal and axeniccultures. Physicol Plant 22:1069-1078

Birkhoff G, Rota GC (1982) Ordinary differential equations. Ginn, Boston

Celik C (2008) The stability and Hopf bifurcation for a predator-prey system with time delay. Chaos Solitons Fractals 37(1):87-99

Chen Y, Yu J, Sun C (2007) Stability and Hopf bifurcation analysis in a three-level food chain system with delay. Chaos Solitons Fractals 31(3):683-694

Das T, Mukherjee RN, Chaudhuri KS (2009) Harvesting of a preypredator fishery in the presence of toxicity. Appl Math Model $33: 2282-2292$
Freedman HI, Shukla JB (1991) Models for the effect of the toxicant in single species and predator-prey system. J Math Biol 30:15-30

Ghosh M, Chandra P, Sinha P (2002) A mathematical model to study the effect of toxic chemicals on a prey-predator type fishery. J Biol Syst 10:97-105

Gopalsamy K (1992) Stability and oscillation in delay-differential equations of population dynamics. Kluwer, Dordrecht

Guo M, Xu X, Li R (2003) Impulsive functional differential inclusions and fuzzy populations models. Fuzzy Sets Syst 138:601-615

Hallam TG, Clark CE, Jordan GS (1983a) Effects of toxicants on population :a qualitative approach II. First-order kinetics. J Math Biol 18:25-37

Hallam TG, Clark CE, Lassiter RR (1983b) Effects of toxicants on population :a qualitative approach I. Equilibrium environmental exposure. Ecol Model 18:291-304

Hallam TG, Luna JTD (1984) Effects of toxicants on population :a qualitative approach III. Environmental and food chain pathways. J Theor Biol 109:411-429

He J, Wang K (2007) The survival analysis for a single-species population model in a polluted environment. Appl Math Model 31:2227-2238

Jensen AL, Marshall JS (1982) Application of a surplus production model to assess environmental impacts on exploited populations of Daphina pluex in the laboratory. Environ Pollut Ser A 28:273-280

Kuang Y (1993) Delay differential equations with applications in population dynamics. Academic Press, New York

Liu WM (1994) Criteria of Hopf bifurcations without using eigen values. J Math Anal Appl 182(1):250-256

Luna JTD, Hallam TG (1987) Effect of toxicants on population: a qualitative approach IV. Resource-consumer-toxicants models. Ecol Model 35:249-273

MacDonald M (1989) Biological delay system: linear stability theory. Cambridge University Press, Cambridge

Maiti A, Pal AK, Samanta GP (2008) Effect of time-delay on a food chain model. Appl Math Comp 200:189-203

Maiti A, Pal AK, Samanta GP (2008) Usefulness of biocontrol of pests in tea: a mathematical model. Math Model Nat Phenon 3(4):96-113

Maynard-Smith J (1975) Models in ecology. Cambridge University Press, Cambridge

Nelson SA (1970) The problem of oil pollution in the sea In: Russell FS, Yonge M (eds) Adv. in Marine Biol. Academic Press, London, pp 215-306

Pal D, Mahapatra GS, Samanta GP (2012) A proportional harvesting dynamical model with fuzzy intrinsic growth rate and harvesting quantity. Pac Asian J Math 6:199-213

Pal D, Mahapatra GS, Samanta GP (2013) Optimal harvesting of prey-predator system with interval biological parameters: a bioeconomic model. Math Biosci 241:181-187

Pal D, Mahapatra GS, Samanta GP (2014) Bifurcation analysis of predator-prey model with time delay and harvesting efforts using interval parameter. Int J Dynam Control

Peixoto M, Barros LC, Bassanezi RC (2008) Predtor-prey fuzzy model. Ecol Model 214:39-44

Pratt R (1940) Influence of the size of the inoculum on the growth of Chlorella vulgaris in freshly prepared culture medium. Am J Bot 27:52-56

Pratt R, Fong J (1940) Studies on Chlorella vulgaris, II. Further evidence that chlorella cells form a growth-inhibiting substance. Am J Bot 27:431-436

Rice EL (1984) Allelopathy, 2nd edn. Academic Press, New York

Rice TR (1954) Biotic influences affecting population growth of planktonic algae. US Fish Wildl Serv Fish Bull 54:227-245 
Samanta GP (2010) A two-species competitive system under the influence of toxic substances. Appl Math Comp 216:291-299

Song Y, Han M, Peng Y (2004) Stability and Hopf bifurcations in a competitive Lotka-Volterra system with two delays. Chaos Solitons Fractals 22(5):1139-1148
Xua R, Gan Q, Ma Z (2009) Stability and bifurcation analysis on a ratio-dependent predator-prey model with time delay. J Comp Appl Math 230:187-203 